nature

medicine

\section{Move against malaria}

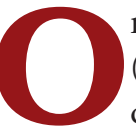
n 2 May, the US Agency for International Development (USAID) endorsed the spraying of the insecticide dichloro-diphenyl-trichloroethane (DDT) inside houses, as part of efforts to control malaria in sub-Saharan Africa. The World Health Organization (WHO) is expected to follow suit when they issue their new guidelines for so-called indoor residual spraying. The significance of this policy change cannot be overestimated. It could be one of the most effective decisions of the past few decades in the battle to halt the tide of malaria and force the epidemic into a retreat.

Indoor spraying with DDT-a compound that both kills and repels the mosquitoes that harbor the malaria parasite- - has been causally associated with declining malaria rates in several countries. In the 1940s, it helped eradicate malaria from the US. Yet since the 1970s, its use has been discontinued in many African countries, largely due to pressure from Europe and the US: places in which malaria is no longer a problem. And, despite efforts from the Roll Back Malaria initiative-a collaboration between more than 90 international organizations including the WHO and the World Bank-to halve the number of deaths by 2010, the incidence of malaria in Africa continues to increase (Lancet 365,1439; 2005).

Why was an insecticide with evident efficacy abandoned? The problem stems from the massive and indiscriminate overuse of DDT for agricultural purposes in the US. In 1972, amid fears of adverse environmental consequences, the US Environmental Protection Agency banned the domestic use of DDT. In 2001, the European Community signed a treaty in Stockholm banning the agricultural use of DDT and recommending its gradual phase-out for public health purposes.

Many African countries depend on donors to fund malaria control, and DDT did not receive support from powerful international funding organizations. The Roll Back Malaria initiative actively discouraged indoor spraying with DDT, choosing instead to support other strategies - mainly the use of (non-DDT) insecticidetreated bednets. Pressure from the international community, as crop exports to the US and Europe were blocked due to traces of DDT, prompted many African countries to ban the compound.

Most of the anti-DDT sentiment has stemmed from valid concerns about the effects of its use on the environment. But DDT has also been more controversially linked to cancer, nervous system defects and other adverse effects on human health. These claims have either been weak and since refuted, or have been linked to the massive agricultural overuse of DDT (see News Feature on page 870). Available evidence suggests that targeted use of DDT for malaria control is unlikely to be toxic — small quantities are sprayed directly onto walls only once per year, making the risk of environmental leakage and human ingestion of the compound low.

So the recent decision to endorse and fund the use of the insecticide specifically for malaria control is welcome news. So far so good. But is it good enough?

Experience has shown that indoor spraying can be effective at reducing the burden of disease, but organizational change and a commitment to sustained and consistent allocation of resources is also essential. Current control strategies-mainly treated bednets-can use existing infrastructure. Indoor spraying, on the other hand, requires strong support from governments and the creation of a centralized infrastructure to properly train personnel, to educate communities, to coordinate spraying and to make sure that coverage is sufficient to make an impact. And, crucially, governments need to ensure that DDT is not diverted for use for agricultural purposes.

Implementing any new strategy in countries with poor infrastructure and decentralized health systems is hard enough. But in the case of DDT, the malaria control effort faces an additional challenge-overcoming the insecticide's toxic image. This means that it is vital that funding is adequate to support monitoring and research into the possible adverse consequences, concomitant with the reintroduction of the insecticide. It is known that DDT accumulates in fat cells and can be found in breast milk, but the consequences of this are not clear. Studies have linked DDT sprayed inside houses with increased premature birth and with a shorter lactation period, but these studies are controversial. Research should therefore be initiated to investigate the effect of DDT on overall infant mortality as well as on malaria-associated infant mortality. Adequate funding will also be required to monitor the emergence of resistance to DDT and to support research into the consequences.

The reintroduction of DDT has the potential to make a marked impact on the global malaria epidemic, but support for the insecticide needs to be strong, and much forward-planning will be required to ensure its safe use and to counter past opposition. Organizations such as USAID and the WHO should ensure that the extent of the funding allocated to both DDT implementation and research is sustained and equal to the daunting challenge that lies ahead. Otherwise a brave new move may fail. 